\title{
Antagonizing SET Augments the Effects of Radiation Therapy in Hepatocellular Carcinoma through Reactivation of PP2A-Mediated Akt Downregulation $\$$
}

\author{
Chao-Yuan Huang, Man-Hsin Hung, Chi-Ting Shih, Feng-Shu Hsieh, Chiung-Wen Kuo, \\ Ming-Hsien Tsai, Shih-Shin Chang, Yung-Jen Hsiao, Li-Ju Chen, Tzu-I. Chao, \\ and Kuen-Feng Chen
}

\begin{abstract}
Division of Radiation Oncology, Department of Oncology, National Taiwan University Hospital and National Taiwan University College of Medicine, Taipei, Taiwan (C.-Y.H.); Division of Medical Oncology, Department of Oncology, Taipei Veterans General Hospital, Taipei, Taiwan (M.-H.H.); Institute of Biopharmaceutical Sciences (C.-T.S.) and School of Medicine (M.-H.H.), National Yang-Ming University, Taipei, Taiwan; Department of Medical Research (F.-S.H., M.-H.T., S.-S.C., Y.-J.H, L.-J.C., K.-F.C.) and National Center of Excellence for Clinical Trial and Research (K.-F.C.), National Taiwan University Hospital, Taipei, Taiwan; Department of Medical Imaging and Radiological Technology, Yuanpei University, Hsinchu, Taiwan (C.-Y.H., C.-W.K.); and SupremeCure Pharma Inc., Taipei, Taiwan (T.-I.C.)
\end{abstract}

Received March 13, 2018; accepted June 13, 2018

\section{ABSTRACT}

Increasing evidence suggests that SET functions as an oncoprotein and promotes cancer survival and therapeutic resistance. However, whether SET affects radiation therapy (RT)-mediated anticancer effects has not yet been explored. We investigated the impact of SET on RT sensitivity in hepatocellular carcinoma (HCC). Using colony and hepatosphere formation assays, we found that RT-induced proliferative inhibition was critically associated with SET expression. We next tested a novel SET antagonist, $\mathrm{N}^{4}-(3-$ ethynylphenyl)-6,7-dimethoxy- $\mathrm{N}^{2}$-(4-phenoxyphenyl) quinazoline2,4-diamine (EMQA), in combination with RT. We showed that additive use of EMQA significantly enhanced the effects of RT against $\mathrm{HCC}$ in vitro and in vivo. Notably, compared with mice receiving either RT or EMQA alone, the growth of PLC5 xenografted tumor in mice receiving $R T$ plus EMQA was significantly reduced without compromising treatment tolerability. Furthermore, we proved that antagonizing SET to restore protein phosphatase 2A-mediated phospho-Akt ( $\mathrm{p}-\mathrm{AKT}$ ) downregulation was responsible for the synergism between EMQA and RT. Our data demonstrate a new oncogenic property of SET and provide preclinical evidence that combining a SET antagonist and RT may be effective for treatment of HCC. Further investigation is warranted to validate the clinical relevance of this approach.

\section{Introduction}

Hepatocellular carcinoma (HCC) is one of the most fatal malignant diseases and is responsible for more than 760,000 deaths worldwide every year (El-Serag, 2011; Forner et al., 2012; Torre et al., 2015). Such a high occurrence of HCCrelated death indicates an unmet medical need calling for exploration and development of novel treatments to improve clinical outcomes. Treating HCC is challenging; the number of patients eligible for curative resection or liver transplantation is limited (El-Serag, 2011), and long-term benefits are largely compromised by a high local recurrence rate (Poon et al., 2002;

This work was supported by grants from the Ministry of Science and Technology of Taiwan [MOST 105-2341-B-002-088-MY3 and 105-2314-B-075067] and Taipei Veterans General Hospital [V105B-015 and V106B-012]; partial support was provided by Taiwan Clinical Oncology Research Foundation and Taipei Veterans General Hospital-National Yang-Ming University Excellent Physician Scientists Cultivation Program.

https://doi.org/10.1124/jpet.118.249102.

S This article has supplemental material available at jpet.aspetjournals.org.
Facciuto et al., 2008). Thus, how to make progress in improving local HCC control and decreasing recurrence is of utmost clinical interest.

Radiation therapy (RT), by transmitting high-energy radiation, could effectively shrink tumors within the irradiated area, and thus serve as a good modality for local control (Yarnold, 1997). Currently, the use of RT, either as the major treatment modality or in combination with other treatments, has been approved in many types of malignant diseases (Ragaz et al., 2005; Corvò, 2007; Tepper et al., 2008; Bonner et al., 2010). However, the use of RT for the treatment of HCC has been far less discussed and explored. Arguments against the use of RT in HCC include a higher risk of RT-induced liver toxicity in patients with cirrhotic background (Hawkins and Dawson, 2006; Klein and Dawson, 2013). Furthermore, occurrence of endogenous radioresistance has been reported in HCC cells. Several different biologic events, including upregulation of AKT- and cancer-stemness-related signaling, were reported to be contributors to the development of radioresistance in

ABBREVIATIONS: 53BP1, p53-binding protein 1; EMQA, $\mathrm{N}^{4}$-(3-ethynylphenyl)-6,7-dimethoxy- $\mathrm{N}^{2}$-(4-phenoxyphenyl) quinazoline-2,4-diamine; HCC, hepatocellular carcinoma; p-AKT, phospho-Akt; PBS, phosphate-buffered saline; PP2A, protein phosphatase 2A; RT, radiation therapy; siRNA, small-interfering RNA. 
HCC cells (Piao et al., 2012; Shimura et al., 2012; Huang et al., 2013). Protein phosphatase 2A (PP2A) is a major serine/ threonine phosphatase that participates in many important cellular functions, including DNA damage repair, cell survival, and cellular transformation (Mumby, 2007; Perrotti and Neviani, 2013). Our group and others have previously characterized the tumor suppressor roles of PP2A in HCC as a negative regulation of many critical oncogenic signals, such as AKT (Chen et al., 2010; Yu et al., 2014; Hung et al., 2016; Ruvolo, 2016). More interestingly, we have shown that aberrant expression of SET, a PP2A inhibitor, promoted the maintenance of HCC cells, and that antagonizing SETmediated PP2A inactivation induced apoptosis of HCC cells (Hung et al., 2016).

SET is a multifunctional oncoprotein involved in a number of important cellular functions (Switzer et al., 2011; Hung and Chen, 2017). Its ability to inhibit PP2A activity is the most well known function of SET (Li et al., 1996), though many recent studies have highlighted the ability of SET to modulate chromatin dynamics, DNA damage responses, and cell cycle progression (Seo et al., 2001; Cervoni et al., 2002; Canela et al., 2003; Kutney et al., 2004; González-Arzola et al., 2015; Kalousi et al., 2015). Through the formation of a complex with TAF- $1 \alpha$ and pp32, SET inhibits acetylation of nucleosomes by binding to and masking histone acetyltransferase targets, thereby altering gene transcription (Seo et al., 2001). SET also modulates the metastatic potential of cancer cells through interaction with nm23-H1 (Fan et al., 2003) and/or Rac1 (Switzer et al., 2011). Importantly, antagonizing aberrant activation of SET also promotes the development of many anti-cancer therapeutics (Cristobal et al., 2015; Rincón et al., 2015); however, the possibility that it influences radiosensitivity has not yet been discussed.

In the present study, we aimed to explore the potential roles of SET associated with the radiosensitivity of HCC. By molecular manipulations, we showed that the expression of SET protein critically affected the RT-induced DNA-damage and apoptosis of HCC. Furthermore, reversing SET-mediated PP2A inhibition was an effective strategy to augment the in vitro and in vivo effects of RT against HCC.

\section{Materials and Methods}

Antibodies and Reagents. Antibodies for immunoblotting of PARP-1 and AKT were purchased from Santa Cruz Biotechnology (Dallas, TX), and anti-p-AKT (Ser473) was from Cell Signaling Technology Inc. (Danvers, MA). Anti-SET was purchased from Bethyl Laboratories (Montgomery, TX) and anti-Actin was from Proteintech (Rosemont, IL). Anti-PP2Ac was obtained from MilliporeSigma (Darmstadt, Germany) and anti-DDK-tag was from Origene (Rockville, MD). Anti- $\beta$-Tubulin was purchased from Epitomics (Burlingame, CA). For immunofluorescence staining, anti-53BP1 antibody (Cell Signaling Technology Inc.), anti-PDI antibody (abcam, Cambridge, MA), and Alexa Fluor 488-conjugated goat anti-rabbit antibody and Alexa Flour 594conjugated AffiniPure Goat Anti-Mouse antibody (Jackson ImmunoResearch Laboratories, Inc., West Grove, PA) were used. For flow cytometry detecting rH2AX phosphorylation, Alexa Fluor 488 anti-H2A. X Phospho (Ser139) Antibody (BioLegend, San Diego, CA) was used. To validate the clinical relevance of targeting SET to enhance the anti-HCC effect of RT, we used a novel compound, EMQA, developed in our laboratory (Hung et al., 2016). For this experiment, EMQA was dissolved in dimethyl sulfoxide and then added to cells in $5 \%$ fetal bovine serum (FBS)-containing medium.
Cell Culture and Western Blot Analysis. The PLC5 and Hep3B cell lines were obtained from American Type Culture Collection (Manassas, VA), the HA22T and HA59T cell lines were obtained from the Bioresource Collection and Research Center (Hsinchu, Taiwan), and the NeHepLXHT cell line was a kind gift from Dr. Jui-Hsiang Hung. All the HCC cells were maintained in Dulbecco's modified Eagle's medium, and the NeHepLXHT cells were kept in F12. All the cells were supplemented with $10 \% \mathrm{FBS}$ and maintained in a humidified incubator at $37^{\circ} \mathrm{C}$ and $5 \% \mathrm{CO}_{2}$ in air. For experiments, cell lysates were collected after exposure to the indicated treatments, and were prepared for immunoblotting and/or coimmunoprecipitation.

Drug and Radiation Treatment of Cells. Irradiation was administrated using a cobalt-60 unit (dose rate of $0.58 \mathrm{~Gy} / \mathrm{min}$ ) with the source-axis-distance set at $80 \mathrm{~cm}$ to the bottom of the flask (CIS Bio International, France). For experiments that combined drugs with radiation, cells were treated with EMQA 1 hour before irradiation.

Cell Proliferation, Apoptosis, and Sphere-Formation Analysis. For colony formation assay, Hep3B cells with or without SETknockdown were seeded in a six-well plate at a density of 1000 cells per well, and the indicated treatments performed 1 day later. The area of HCC colonies formed was assessed 12 days later by fixing with $4 \%$ paraformaldehyde solution and staining with $0.5 \%$ crystal violet. The extent of apoptotic cell death was determined by two other methods: flow cytometry (sub-G1 assay) and Western blot for poly (ADP-ribose) polymerase cleavage. For the generation of hepatospheres, $1 \times 10^{3} \mathrm{HCC}$ cells were plated per well of a six-well ultra-low attachment plate (Corning, New York, NY), and grown in serum-free medium with B-27, human epidermal growth factor, and basic fibroblast growth factor. After 2 weeks, the number and size of hepatospheres formed were determined.

Immunofluorescence. Cells $\left(2.5 \times 10^{5}\right)$ were seeded on $60-\mathrm{mm}$ dishes with coverslips, cultured for 24 hours, and then received RT and/or EMQA treatment. After indicated treatments, cells were fixed with $4 \%$ paraformaldehyde, followed by permeabilization with $0.3 \%$ Triton X-100/phosphate-buffered saline (PBS) for 15 minutes, and blocking with $5 \%$ bovine serum albumin (BSA)/PBS for at least 1 hour at room temperature. The specimen was incubated with antibody dilution buffer (1\% BSA/0.3\% Triton X-100/PBS) containing primary antibody overnight at $4^{\circ} \mathrm{C}$. Cells were washed with PBS containing $0.05 \%$ Tween-20 three times and then incubated with fluorochromeconjugated secondary antibody for 1 hour at room temperature. DAPIFluoromount-G (SouthernBiotech, Birmingham, AL) was used to highlight the nuclei of cancer cells. Cell images were acquired by fluorescence microscope (Axio Imager A1; Zeiss, Oberkochen, Germany). The average numbers of p53-binding protein 1 (53BP1) foci were determined by MetaMorph software version 7.7.7.0 (Molecular Devices, San Jose, CA) with Granularity Application Module.

H2A.X Phosphorylation. H2A.X phosphorylation was analyzed by flow cytometry (Muslimovic et al., 2008). In brief, cells were collected after treatment, suspended, and incubated with $5 \mu$ l Alexa Fluor 488 anti-H2A.X Phospho (Ser139) antibody (BioLegend) and incubated in the dark for 1 hour at $4^{\circ} \mathrm{C}$. Afterward, cells were resuspended and analyzed by flow cytometry.

Comet Assay. Huh7 cells with or without SET-silencing were seeded on 100-mm dishes at a concentration of $1 \times 10^{5}$ cells per dish and exposed to irradiation (10 Gy) 1 day before being examined by comet assay (Trevigen's Comet Assay kit; Gaithersburg, MD) performed according to the manufacturer's instructions. Briefly, 0.5 hours after irradiation, cells were collected, mixed with LMAgarose, and spread on specific slides (CometSlides; Trevigen). After the agarose solidified, the slides were placed in the lysis solution at $4^{\circ} \mathrm{C}$ for 30 minutes. Slides were then subjected to electrophoresis $(1 \mathrm{~V} / \mathrm{cm}$ for 45 minutes) at $4^{\circ} \mathrm{C}$ in the dark. After electrophoresis, cells were immersed in DNA Precipitation Solution for 30 minutes, fixed with $70 \%$ ethanol, and stained with DAPI. Nuclei were visualized using fluorescence microscopy. DNA damage (tail moment) was quantified using CaspLab software (casplab.com) (Końca et al., 2003). For each experiment, more than 50 cells were quantified and analyzed. 
PP2A Phosphatase Activity. The PP2A activities in cancer cells and tumor tissues were determined using the PP2A activity kit obtained from Upstate Biotechnology Inc. (Lake Placed, NY) and carried out as previously described (Hung et al., 2016).

Plasmids, Small-Interfering RNA, Cell Transfection, and Lentiviral Transductions. The cDNA of AKT and SET were purchased from Origene. Smart-pool small-interfering (si)RNA against PP2Ac (L-003598-01) and control (D-001010-10) were all purchased from Dharmacon (Chicago, IL), and the short-hairpin (sh)RNA against SET and empty vector (pLKO.1) was obtained from the National RNAi Core Facility platform (Taipei, Taiwan). For transient expression experiments, plasmids were pretransfected with Lipofectamine 2000 (Invitrogen/ Thermo Fisher Scientific, Waltham, MA), and siRNA was pretransfected with the DharmaFECT4 Transfection Reagent (Dharmacon) for 24 hours, then underwent radiation and drug treatment for another 24 hours. On the other hand, cells were transduced with shRNA lentivirus constructs by polybrene and selected by puromycin.

Xenograft Tumor Growth. Male Ncr athymic nude mice (5-7 weeks of age) were obtained from the National Laboratory Animal Center (Taipei, Taiwan). All experimental procedures using these mice were performed in accordance with protocols approved by the Institutional Laboratory Animal Care and Use Committee of National Taiwan University. Each mouse was inoculated subcutaneously in the dorsal flank with $1 \times 10^{6}$ PLC5 cells suspended in $0.1 \mathrm{ml}$ of serum-free medium containing $50 \%$ Matrigel (BD Biosciences, Bedford, MA). When tumors reached $400-600 \mathrm{~mm}^{3}$, mice were randomly assigned to each of the investigational treatments. For the radiation arms, mice were immobilized in a customized harness that exposed the right leg while the remainder of the body was shielded by five times the half-value thickness of lead during radiation. In total, the tumors were irradiated using a cobalt-60 unit with $20 \mathrm{~Gy} \mathrm{(5} \mathrm{Gy/d}$ for 4 days, at a dose rate of $0.5 \mathrm{~Gy} / \mathrm{min}$ ). After 4 days irradiation, mice in the combination treatment arm and the EMQA-alone arm received EMQA at $5 \mathrm{mg} / \mathrm{kg} / \mathrm{d}$ PO daily, whereas the mock-treated mice received vehicle consisting of $0.5 \%$ methylcellulose and $0.1 \%$ polysorbate 80 in sterile water. After this experiment was completed, all the xenografted tumors were resected, measured for net weight, and analyzed by Western blot, p-Akt ELISA (R\&D, Minneapolis, MN), and PP2A activity.

Patient Samples and Immunohistochemical Staining. This study was approved by the ethics committee of the Institutional Review Board of Changhua Christian Hospital. All patients who donated their tumor in the present study signed an approved informed consent at the time of donation in accordance with the Declaration of Helsinki. Tumors were analyzed by immunohistochemical stain for the expression of SET (A302-262A; Bethyl Laboratories) and representative images are shown. The procedure of immunohistochemical staining were conducted as previously described (Yu et al., 2014).

Statistical Analysis. Comparisons of means were analyzed using the independent sample $t$ test by SPSS software for Windows version 17.0 (Chicago, IL).

\section{Results}

Novel Oncoprotein SET Attenuates the Effects of Radiation Therapy against HCC. Higher expression levels of SET oncoprotein were observed in both clinical tumor samples [Fig. 1A; also see Supplemental Fig. 1A for the immunohistochemistry results available from the Human Protein Atlas] and liver cancer cell lines (Supplemental Fig. 1B). To understand whether and how the expression of SET in cancer cell affects its sensitivity to RT, we tested the effects of RT on HCC cells with different SET expression. Stable knockdown of SET in HCC cells was achieved by lentivirus-mediated shRNA delivery. Two separate shRNA target sequences against $S E T$ gene, as well as their knockdown efficiencies at the protein level in two HCC cell lines, Hep3B and PLC5, are shown in Fig. 1B. Tumor sphere formation assay was then used to evaluate the anchorageindependent growth abilities of cancer cells with/without SETknockdown under RT treatment. As shown in Fig. 1C, RT reduced hepatosphere formation in Hep3B cells, and, notably, SET-knockdown further augmented the effects of RT in these cells. By contrast, RT-induced reduction of hepatosphere formation was abolished by SET-overexpression (Supplemental Fig. 1C). Furthermore, using a colony formation assay, we showed that RT-induced proliferative inhibition was more prominent in PLC5 cells with SET-knockdown (Fig. 1D). Taken together, the results above suggest that the expression of oncoprotein SET determines the radiosensitivity of HCC cells.

With the knowledge that the basis of RT-induced tumor growth inhibition is its ability to cause complex DNA doublestrand breaks, we next examined whether expression of SET was linked to the degree of RT-mediated DNA damage (Oike et al., 2016). In this study, we analyzed the generation of foci of p53-binding protein 1 , which is a well known protein sensor of DNA damage (Huyen et al., 2004), to highlight the impact of SET expression on the process of DNA damage repair after exposure to RT. Our data showed that 53BP1 foci presenting in cells without RT were very few in number, but the number rapidly increased after exposure to $\mathrm{RT}$, which represented the extensive DNA breaks induced by RT (Fig. 2A). Several hours after radiation exposure, the DNA repair process began, the number of 53BP1 foci decreased, and it took about 24 hours for the cancer cells to reach a level similar pre-RT status, which suggested that DNA damage repair had been completed. Different from control Huh7 cells, the DNA damage repair process was significantly delayed in SET-silenced cells. As shown in the lower panel of Fig. 2A, the foci of 53BP1 remained prominent in cancer cells even at the 24 th hour after radiation exposure. On the other hand, overexpression of SET had minimal effects on the radiation-induced DNA damage and repair process. The kinetics of 53BP1 foci after RT in SEToverexpressing cells were quite similar to control cells (Fig. 2B). We used comet assay next to characterize the extent of global DNA damage induced by RT. In line with the results obtained by 53BP1 foci, SET-knockdown significantly increased the degree of RT-induced DNA damage in HCC (Fig. 2C). Taken together, these results suggest that SET has a critical role in affecting the antitumor effect of RT in HCC.

Antagonizing SET Enhances the Anti-HCC Effect of RT. On the basis of the data above, we hypothesized that antagonizing SET could augment the effects of RT against HCC. To test this hypothesis, we used a novel SET antagonist, EMQA [developed and characterized in our previous work (Chen et al., 2012; Hung et al., 2016)], and tested its effects in combination with RT in HCC. In line with the results using molecular knockdown of SET as shown in Figs. 1 and 2, we found that EMQA significantly potentiated the effects of RT in HCC (Fig. 3). Compared with cells exposed to RT alone, RT plus EMQA significantly reduced the number of tumor spheres (Fig. 3A) and colony-forming ability (Fig. 3B) of HCC. Subsequently, we used sub-G1 analysis and examined the generation of 53BP1 foci to investigate the radiosensitizing effect of EMQA. As shown in Fig. 3C, EMQA promoted RT-induced apoptosis in different HCC cell lines. Moreover, compared to solvent-treated cells, the numbers of 53BP1 foci (Fig. 3D) and the degree of rH2AX (Fig. 3E) in cells pretreated with EMQA persisted much longer after irradiation. Taken together, these data indicate that EMQA synergizes with RT 
A

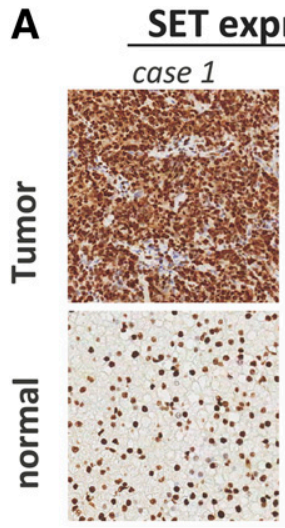

B
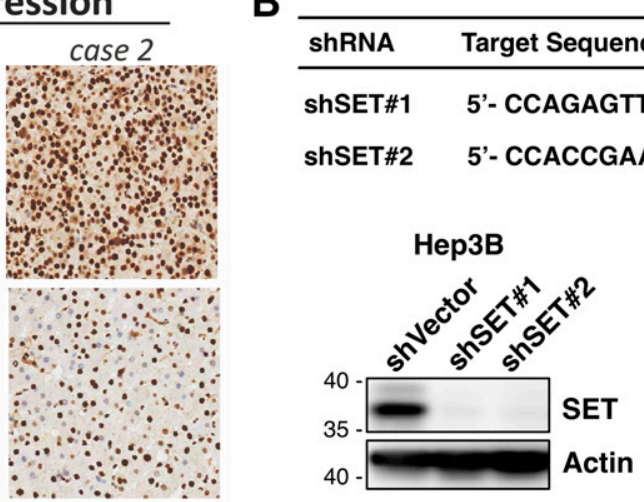

C
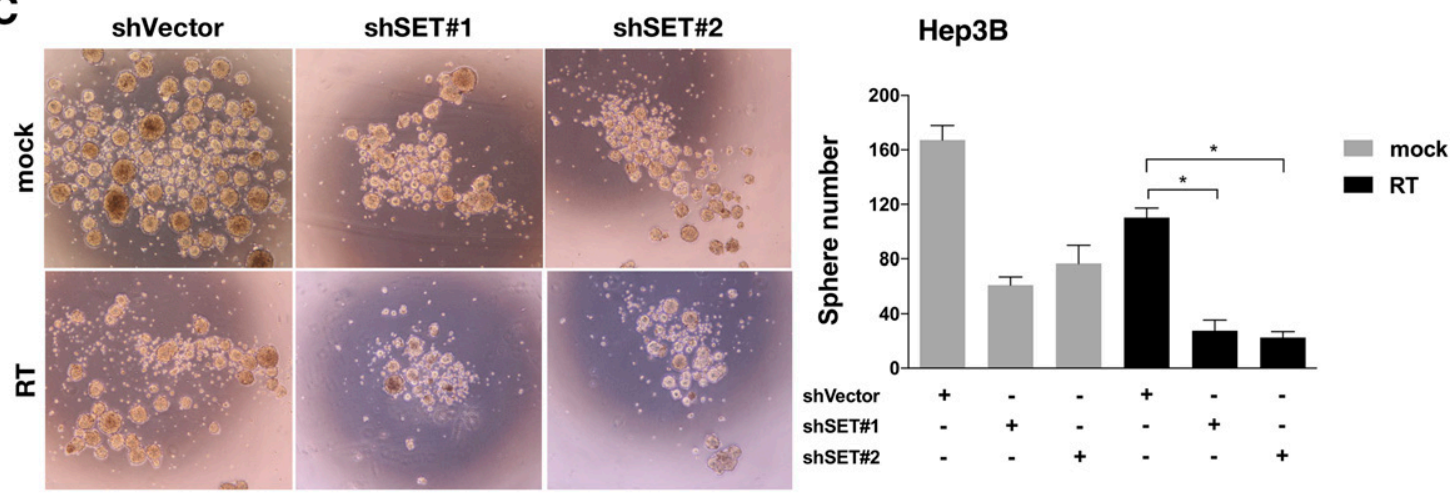

D
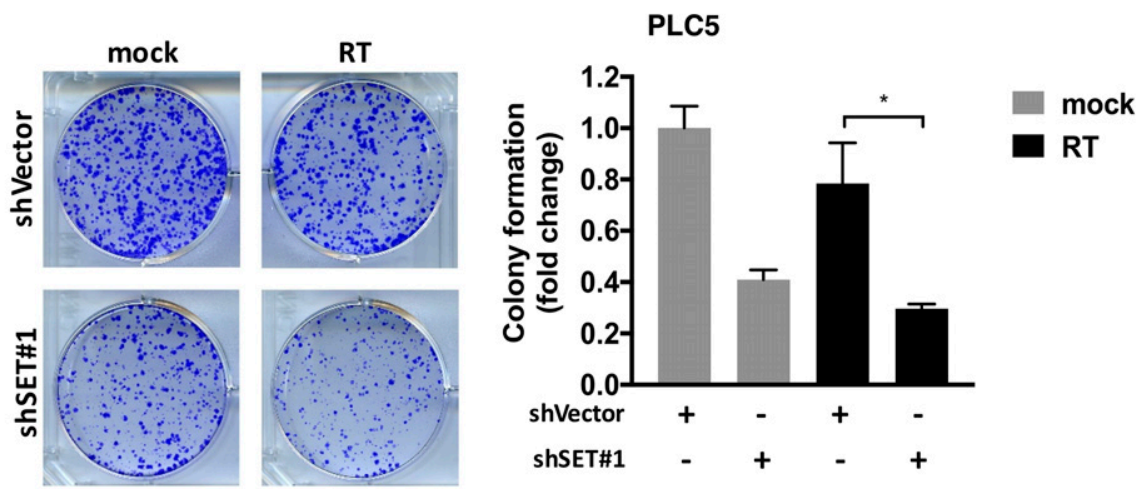

Fig. 1. The oncoprotein SET determines the radiosensitivity of HCC cells. (A) SET protein is highly expressed in HCC clinical samples. The expression of SET was determined in clinical tumor tissues by immunohistochemical stain. (B) The target sequences of shRNA against SET used in this study. Target site refers to the location of the shRNA target sequence in the coding sequence of SET isoform 1. Lower panel, Western blot analysis showing shRNA-knockdown efficiency in Hep3B and PLC5 cells. (C and D) SET silencing augments the RT-mediated growth-inhibitory effects in HCC. The effects of radiotherapy ( $2 \mathrm{~Gy}$ ) on HCC cells with/without SET-knockdown were determined by sphere formation (C) and colony formation assay (D). $N=3$. Representative images of tumor sphere and colony were presented in the left panel and the quantified results were presented over the right panel. Bar: mean; error bar, S.D. $* P<0.05$.

to induce DNA damage and promote apoptotic death of HCC cells.

Synergistic Effect of EMQA Plus RT Is through Reversal of SET-Mediated PP2A Inactivation. According to the literature, SET may be present in and interacts with various factors in different cellular components; for instance, SET forms a complex with NM23-H1 and pp32 in the endoplasmic reticulum (Fan et al., 2003), it inhibits PP2A in cytoplasm (Arif et al., 2014), and it interacts with KAP1 in the nucleus (Kalousi et al., 2015). To understand the underlying mechanism responsible for the sensitizing effects of EMQA on $\mathrm{RT}$, we used an immunofluorescence stain to trace the location of SET after RT and/or EMQA treatment. As shown in Supplemental Fig. 2, SET expression was found principally in the nucleus and cytoplasmic region, whereas evidence for SET in endoplasmic reticulum (as assessed by colocalized with PDI) was not compelling. After treating cells with RT and/or EMQA, the pattern of SET localization did not change significantly. We analyzed HCC cells next by Western blot. As shown in Fig. 4A, downregulation of p-AKT occurred in all the HCC cell lines treated with EMQA plus RT. Since AKT is a direct target of PP2A and EMQA is a SET antagonist that reactivates PP2A by targeting the SET-PP2Ac interaction, we reasoned that EMQA induces PP2A-mediated p-AKT 

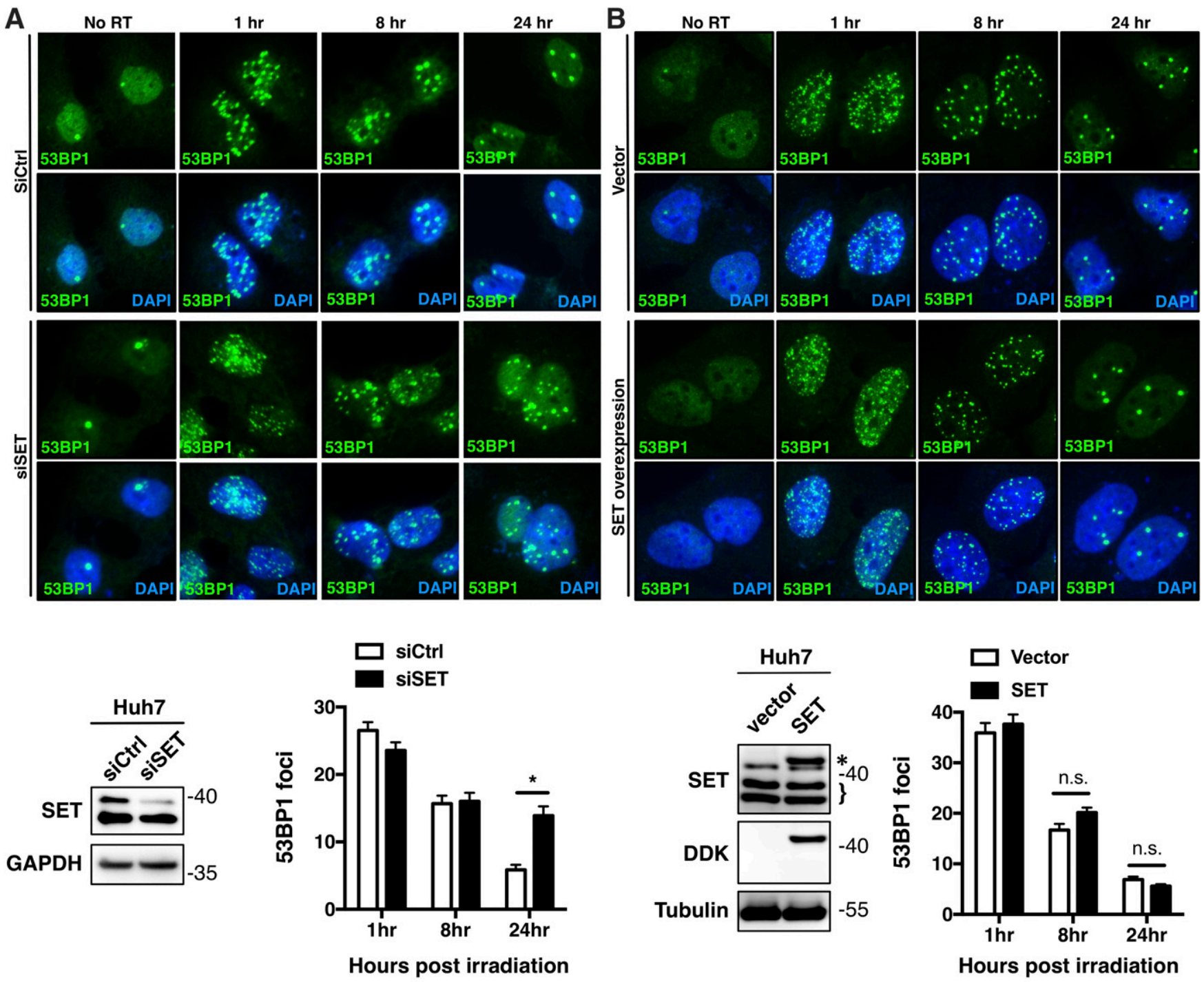

C
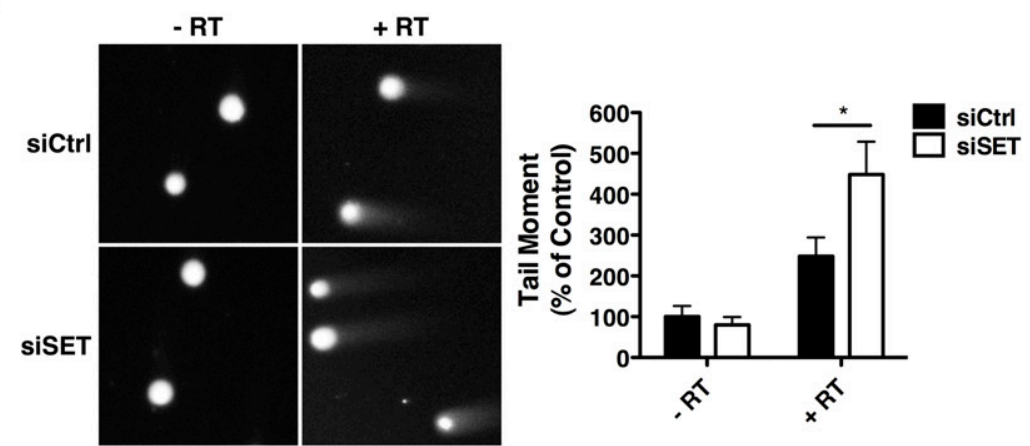

Fig. 2. The effect of SET expression on RT-induced DNA damage in HCC. (A and B) SET-silencing delays the postirradiation DNA repairing process, whereas overexpression of SET has minimal effects on repair of radiation-induced double-strand breaks (DSB) in HCC cells. Huh7 cells with SETknockdown or overexpression were exposed to gamma irradiation (4 Gy) and immunostained for 53BP1 foci (green) at 1, 8, or 24 hours after irradiation. Representative images were shown in the upper panel. Nuclei were stained with DAPI (blue). SET protein expression was confirmed by Western blot. Curly bracket highlights the bands of endogenous SET, and the asterisk indicates the ectopic expressed band. Average numbers of 53BP1 foci for at least 30 nuclei were plotted in the lower right panel. Columns, mean; bars, S.E.M. $* P<0.05$. n.s., not significant. (C) Knockdown of SET enhances the effects of RT to induce DNA damage. Global DNA damage of Huh7 cells after irradiation was examined by single-cell gel electrophoresis (comet assay). Left, representative results of single-cell gel electrophoresis assay in siCtrl-Huh7 and siSET-Huh7 cells. Quantification of comet tails was done using CaspLab software and shown as a bar graph in the right panel (more than 50 cells in each treatment group were examined). Columns, mean; bars, S.E.M. $* P<0.05$.

downregulation to enhance the effects of RT in HCC cells. To test our hypothesis, we first confirmed the mechanism of action of EMQA in HCC cells. Via coimmunoprecipitation and
PP2A activity, we showed that EMQA interfered with the interaction of SET and PP2Ac (Fig. 4B) and increased the activity of PP2A in HCC cells (Fig. 4C). More importantly, 
A
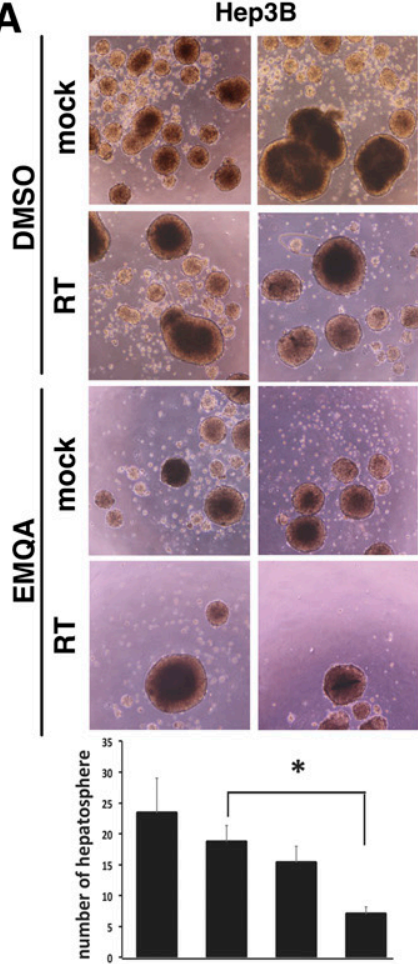

RT

EMQA
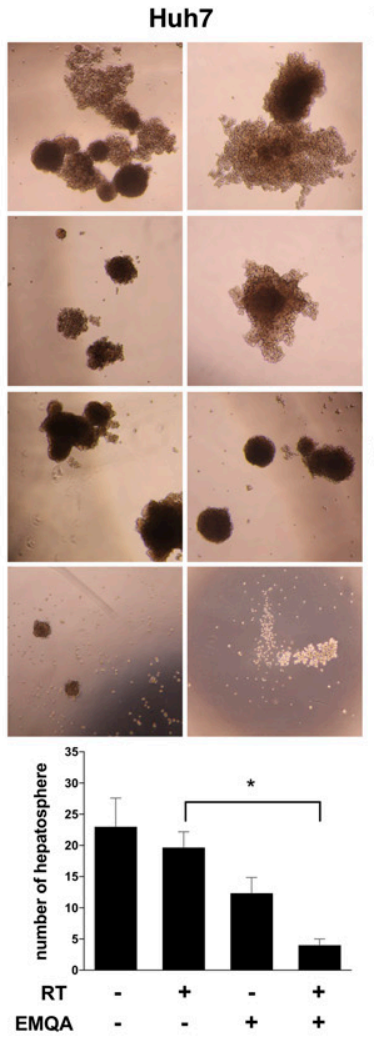

$B_{\text {PLCS }}$

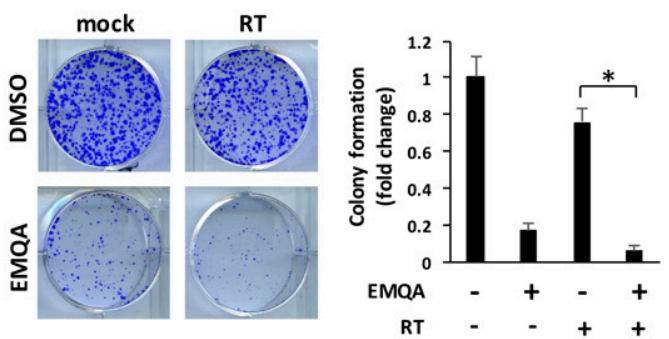

C
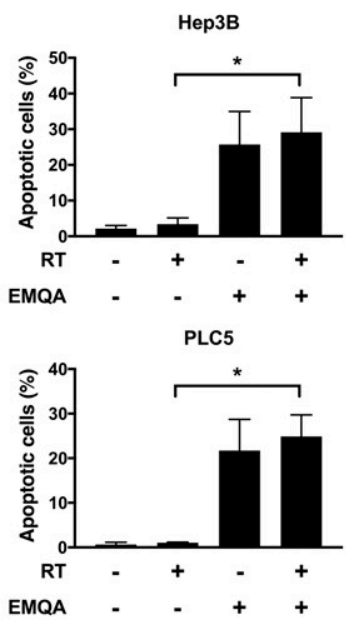

D

Huh7

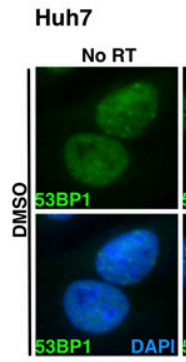

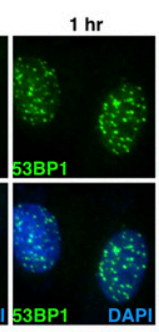

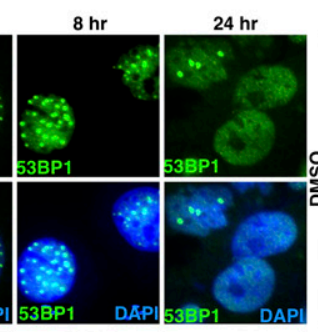

Нер3в
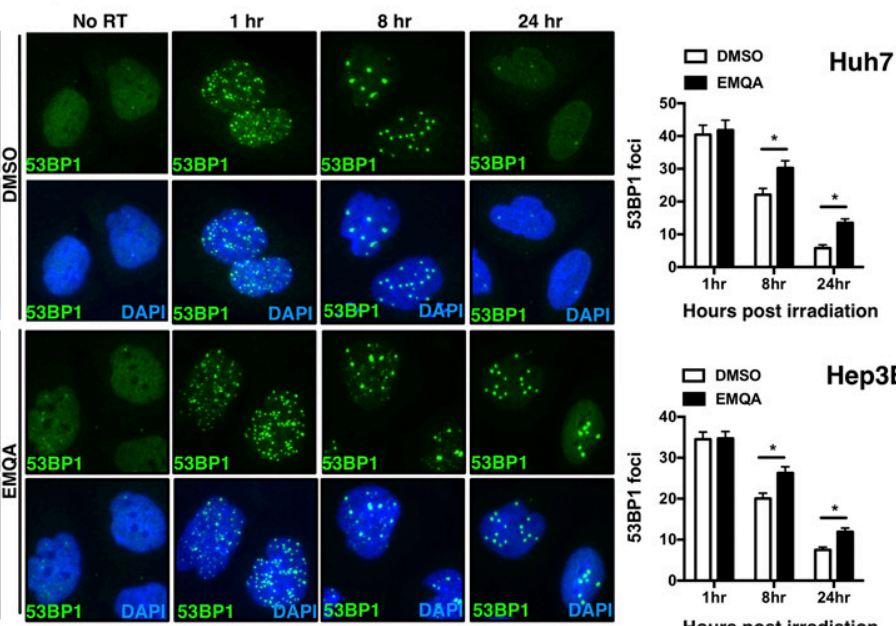

Hours post irradiation

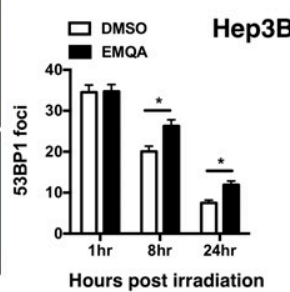

$\mathbf{E}$
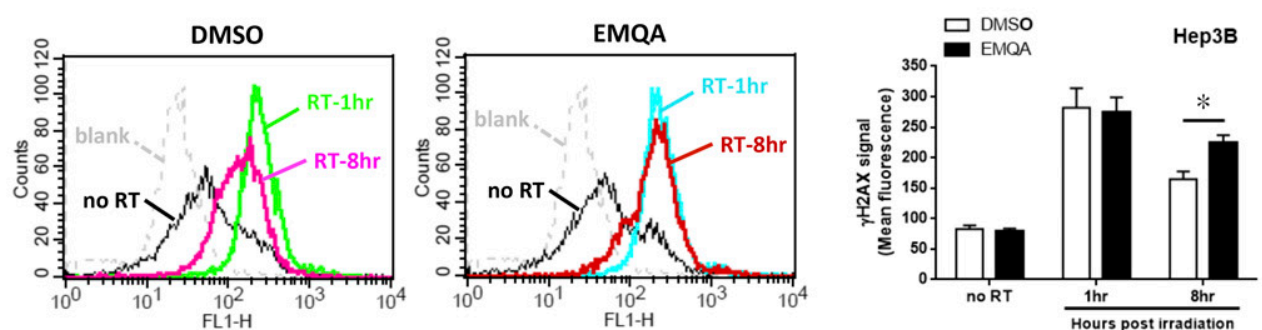

Fig. 3. SET antagonist augments the anti-HCC effects of RT. (A) Representative images and quantitative analysis of the sphere formation of Hep3B and Huh7 cells under indicated treatments. Cells were irradiated with $2 \mathrm{~Gy}$ of IR after EMQA treatment $(1 \mu \mathrm{M})$. $N=3$. Bar, mean; error bar, S.D. $* P<0.05$. (B) Colony formation of PLC5 cells under indicated treatments ( 2 Gy irradiation; EMQA $2 \mu \mathrm{M}) . N=3$. Bar, mean; error bar, S.D. $* P<0.05$. (C) HCC cells were exposed to indicated treatment (2 Gy irradiation; EMQA $2.5 \mu \mathrm{M}$ ) and harvested 24-48 hours after treatment followed by sub-G1 analysis. Bar, mean; error bar, S.D. $* P<0.05$. (D and E) EMQA delays the postirradiation DNA repair progress of HCC cells. The ability of DNA damage repair after 


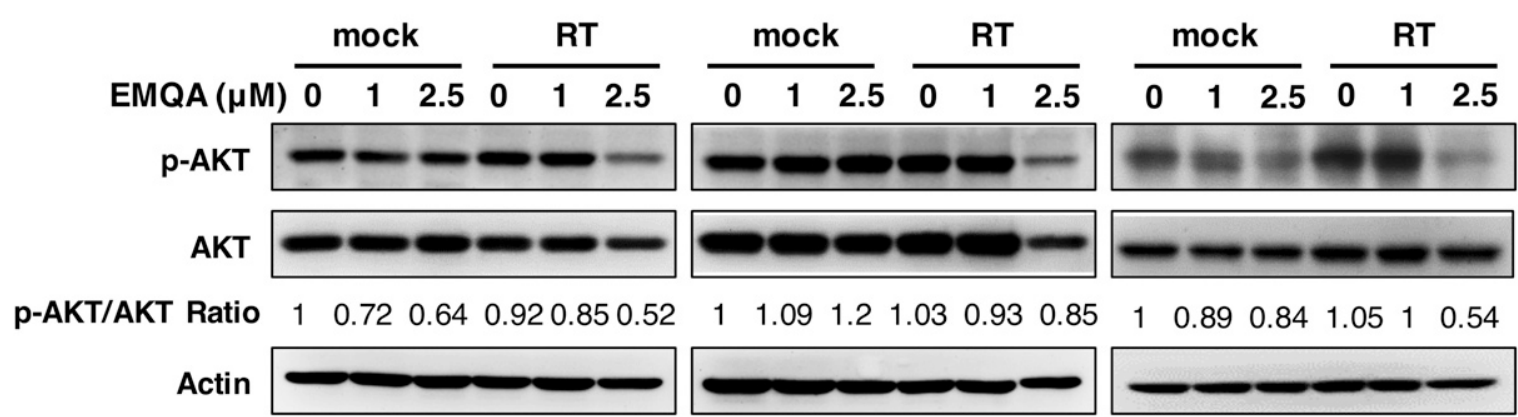

B

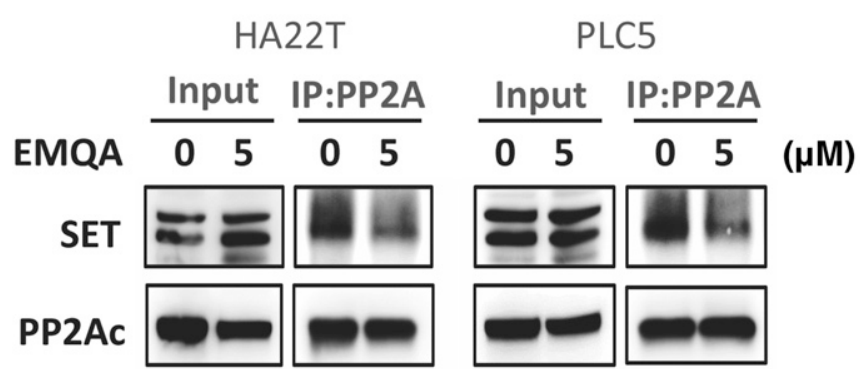

C

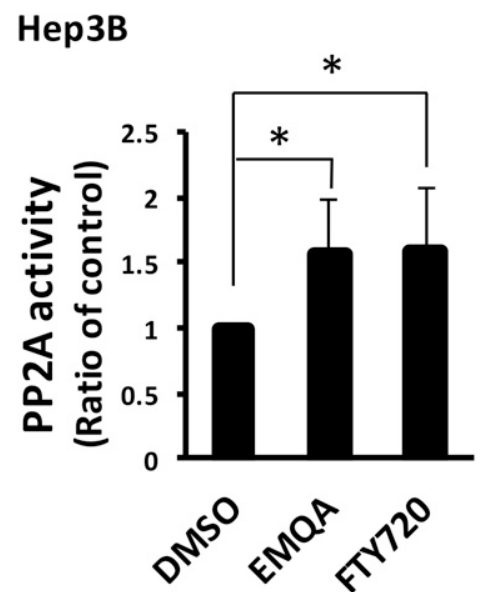

D

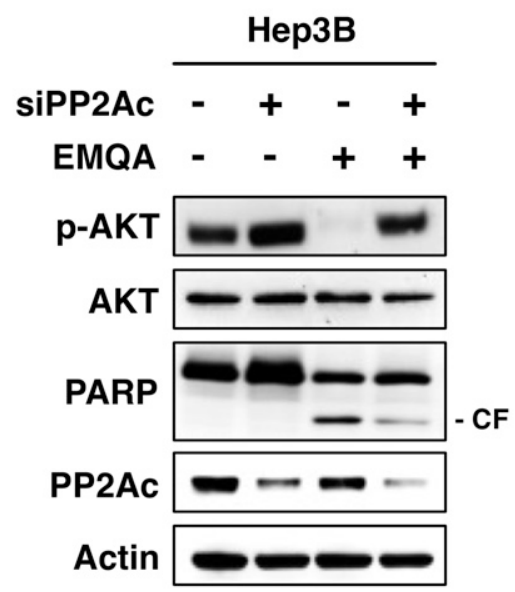

Fig. 4. EMQA dissociates SET-PP2Ac and induces downregulation of p-AKT to enhance RT in HCC. (A) The effects of EMQA plus RT on inducing downregulation of p-AKT of HCC cells. Representative images of Western blot of a panel of HCC cells under indicated treatments. (B) EMQA treatment dissociates SET-PP2Ac binding in HCC. Coimmunoprecipitation was used to detect the association of SET and PP2Ac. (C) PP2A activities in HCC cells were examined after exposure to EMQA $(5 \mu \mathrm{M})$. FTY720 $(10 \mu \mathrm{M})$, a known PP2A activator, served as a positive control. $N=3$. Columns, mean; bars, S.D. $* P<0.05$. (D) Knockdown of PP2Ac abolishes the effects of EMQA on inhibition of p-AKT and promotion of apoptosis in HCC. Representative images of Western blot are shown here. CF, cleaved fragment.

downregulation of p-AKT and the proapoptotic effects induced by EMQA treatment were diminished by knockdown of PP2Ac (Fig. 4D). We performed serial knockdown and/or overexpression experiments next to confirm the roles of the major players mediating the therapeutic effects of RT plus EMQA. First, we generated cancer cells with ectopic expression of myc-tagged AKT by transient transfection, and treated them with EMQA and RT. Compared with wild-type cells, the percentage of subG1 cells induced by RT-EMQA combination treatment was significantly reduced in the AKT-overexpressing cells (Fig. 5A). Next, we generated PP2Ac-knockdown cells and exposed them to EMQA and RT. Importantly, we found that the effects of combination treatment were significantly diminished in PP2Ac-knockdown cells (Fig. 5B). Finally, we sought

exposure to RT was determined by examining the presence of 53BP1 foci (D) and rH2AX (E). Huh7 and Hep3B cells were treated with EMQA (1 $\mu \mathrm{M})$ just before 4-Gy gamma irradiation. Afterward, cells were either immunostained for 53BP1 foci (D) or examined by flow cytometry for the degree of rH2AX (E). For both subfigures, representative images were shown on left and quantitated results of triplicate tests were shown on right. Columns, mean; bars, S.E.M. $* P<0.05$. 

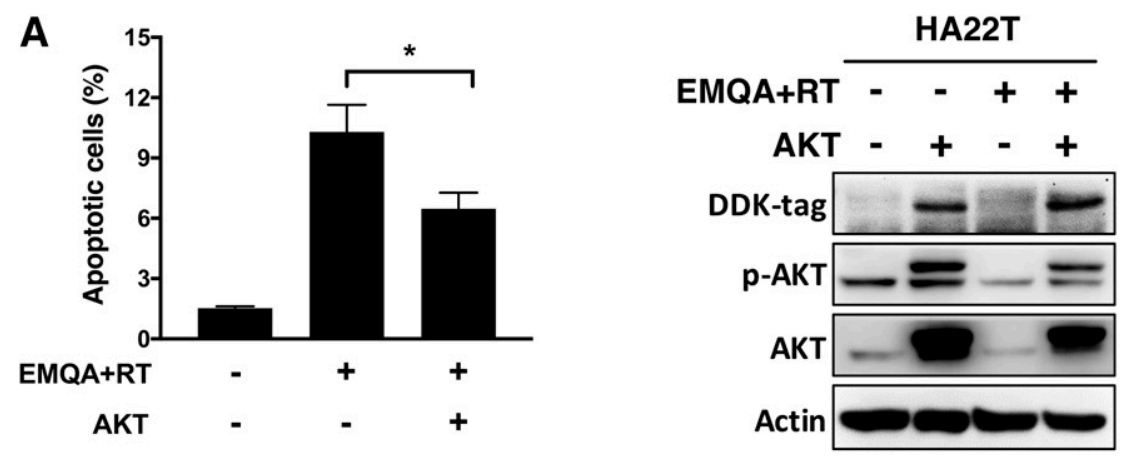

B
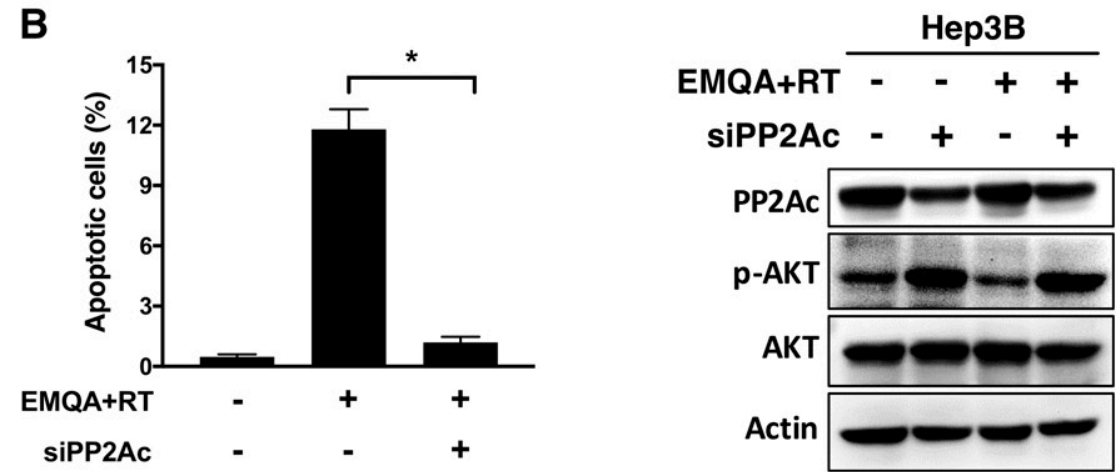

C
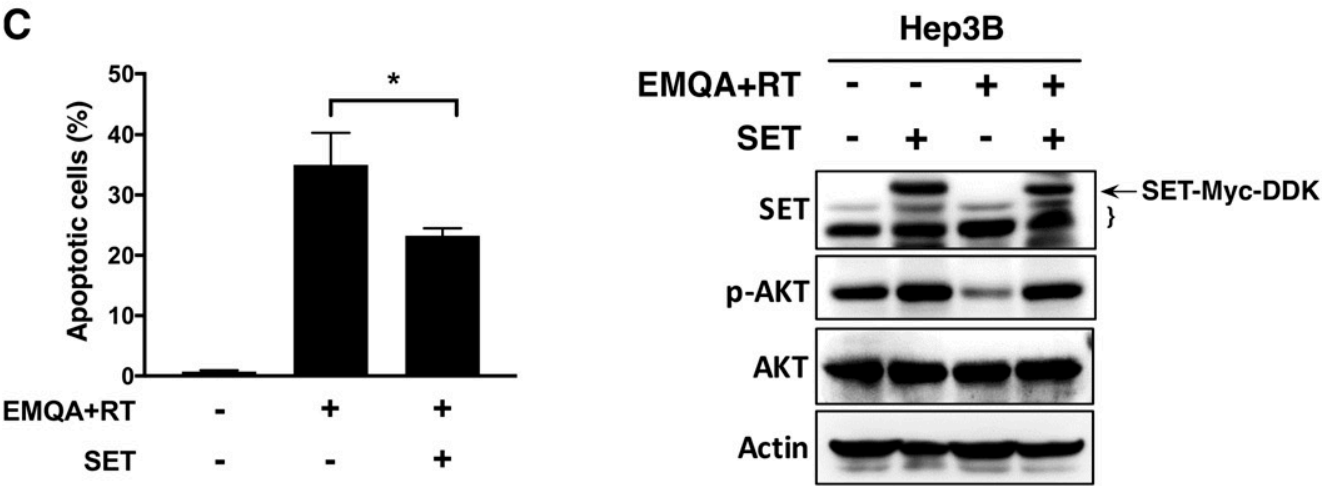

D

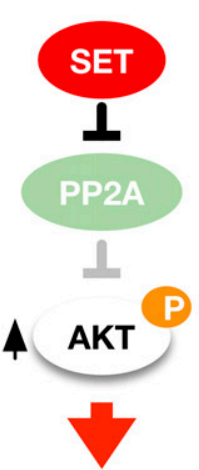

Radiation Resistance

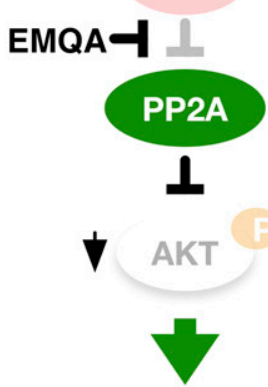

Restore

Radiosensitivity

Fig. 5. SET/PP2Ac/p-AKT signaling determined the effects of RT-EMQA combination treatment in HCC. (A) Ectopic expression of AKT diminishes the effects of RT plus EMQA. After transfection of HA22T cells with AKT-Myc-DDK or empty vector control for 24 hours, cells were treated with RT 2-Gy followed by EMQA $(5 \mu \mathrm{M})$ for another 24 hours and analyzed by flow cytometry (sub-G1, left panel) and Western blot (right panel). Bar, mean; error bars, S.D. $N=3 . * P<0.05$. (B) Downregulating PP2Ac by siRNA reverse the proapoptotic effects of EMQA-RT combination. Hep3B cells were first transfected with PP2Ac siRNA or nontargeting control siRNA for 48 hours and exposed to RT and EMQA. Cell apoptosis was analyzed by flow cytometry (left panel) 
to confirm the roles of SET and showed that the extent of cell apoptosis induced by EMQA-RT combined treatment was significantly decreased in SET-overexpressed HCC cells (Fig. 5C) The above data confirmed that upregulation of PP2A-mediated p-AKT inactivation through antagonizing SET-induced PP2A inactivation is responsible for the synergism between EMQA and RT in HCC (Fig. 5D).

EMQA Enhances the In Vivo Anti-HCC Effects of RT. To understand the clinical potential of using SET as a drug target to augment the effects of RT in HCC patients, we established a preclinical PLC5 xenograft model to test the effects of EMQA and/or RT in vivo. As shown in Fig. 6A, both RT and EMQA demonstrated certain in vivo activity against HCC. But, compared with single treatment, EMQA plus RT led to the most significant inhibition of the average tumor growth without affecting tolerability (Fig. 6, A and B). In agreement, the mean tumor weight of mice receiving combined treatment was significantly lower than the other treatment arms (Fig. 6C). To further elucidate the molecular events responsible for this synergistic effect, we examined the expression of p-AKT and SET and PP2A activity in the tumor extract (Fig. 6, D-F). In line with the results of in vitro work, EMQA plus RT led to downregulation of p-AKT and PP2A reactivation in these xenografted tumors. In conclusion, the results of these in vivo experiments suggest that application of a SET antagonist, like EMQA, has potential to enhance the effects of RT for the treatment of HCC, and that reversing SET-mediated PP2A inactivation in HCC tumor is the major mechanism responsible for such synergism. Further investigation is required to explore the clinical benefit of this novel approach.

\section{Discussion}

In this study, we investigated the impact of a novel oncoprotein, SET, on the radiosensitivity of HCC cells (Figs. 1 and 2) and provided detailed preclinical evidence showing that antagonizing SET-mediated PP2A inactivation augments the anti-HCC effects of RT in vitro and in vivo (Figs. 3-6). Our results not only increase current understanding of the biology of radioresistance in HCC but also demonstrate the potential of using a SET antagonist to improve the effects of RT in HCC. To the best of our knowledge, this is the first work discussing the potential impact of SET protein on radiosensitivity in HCC.

SET is a novel oncoprotein that affects the behavior of cancer cells in multiple ways (von Lindern et al., 1992; Al-Murrani et al., 1999; Fan et al., 2003; Arnaud et al., 2011; Switzer et al., 2011; Hung et al., 2015, 2016). Initially, set gene was identified as a novel fusion partner of a putative oncogene, can, in a patient with acute undifferentiated leukemia (von Lindern et al., 1992; Adachi et al., 1994). Later, Dr. Damuni's group purified this oncoprotein from bovine kidney and characterized its function as a potent inhibitor of PP2A (Li et al., 1995). To date, aberrant expression of SET protein has been reported in patients with various types of malignancies, such as leukemia, sarcoma, and tumors of breast, colon, liver, and lung (Li et al., 1996; Carlson et al., 1998; Chae et al., 2012; Cristobal et al., 2012, 2015; Hung et al., 2015, 2016; Liu et al., 2015). The oncogenic role of SET in HCC was first suggested by Fukukawa et al. (2000). Using a diethylnitrosamineinduced HCC model, they showed that the expression of SET protein in liver tissues was significantly upregulated along with the progression of hepatocarcinogenesis, which indicated that SET may be involved in the development of HCC. Our group, on the other hand, looked at the impact of SET activity on the maintenance of cancer cells (Hung et al., 2016). Using a full panel of HCC cell lines, we showed that the numbers of cancer cell colonies and tumor spheres were significantly reduced by SET knockdown; in contrast, overexpression of SET promoted the growth of HCC. More interestingly, our group and others have found that the activity of SET is associated with the development of resistance to standard anticancer drugs, namely chemotherapies or target therapies, in various malignant diseases (Agarwal et al., 2014; Cristobal et al., 2015; Hung et al., 2015, 2016; Zhang et al., 2015). For example, ectopic expression of SET diminished the effects of paclitaxel against non-small cell lung cancer (Hung et al., 2015) and oxaliplatin against colon cancer (Cristobal et al., 2015). The present study elaborated the impact of SET dysregulation in relation to $\mathrm{RT}$, another important anticancer modality, thus adding to current knowledge. In line with our impression that SET promotes resistance to therapy, we showed that overexpression of SET impaired the radiosensitivity of HCC cells (Supplemental Figure 1C), whereas antagonizing SET by molecular knockdown augmented the effects of RT against HCC in vitro (Fig. 1, C and D; Fig. 2). Furthermore, we provided evidence showing the radiosensitizing effects of a novel SET antagonist, EMQA, in vitro and in vivo in HCC (Figs. 3-5). Interestingly, in addition to our finding emphasizing the radiosensitizing effect mediated by SET/ PP2A/p-AKT signaling, SET is known to interact with DNA damage response (Kalousi et al., 2015; Hung and Chen, 2017), which may also contribute to the synergistic effects of RT and SET antagonist. On the basis of the knowledge that SET overexpression is a recurrent event in HCC and other malignant diseases, targeting SET to enhance the antitumor effects of RT may be an effective therapeutic strategy for the disease. More studies are certainly required to validate this assumption.

In the present work, we showed that activation of AKT signaling may be an important factor contributing to the generation of radioresistance in HCC cells. A similar observation has been made by Dr. Fukumoto's group, who suggested that activation of the AKT/cyclin D1/Cdk4 signaling pathway explained the generation of acquired radioresistance in a small subgroup of CD133-positive cancer cells (termed "cancer stem cells") (Shimura et al., 2012). Our data further demonstrates that dysregulation of SET/PP2A/AKT signaling may affect the susceptibility to RT in all HCC cells and was not limited to CD133positive cells (Figs. 3-6). Inhibition of PP2A is one of the major

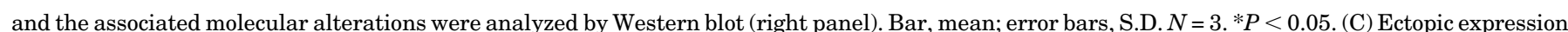

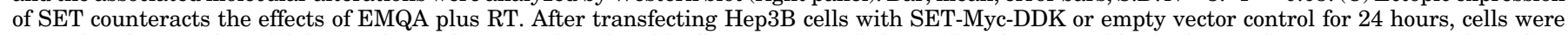

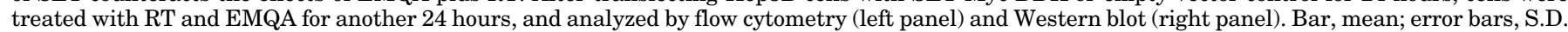

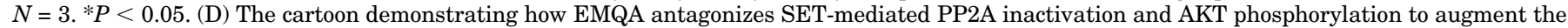
effects of RT. 
A

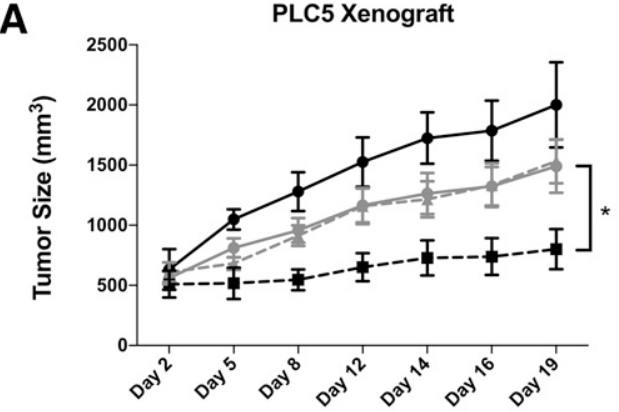

Treatment duration
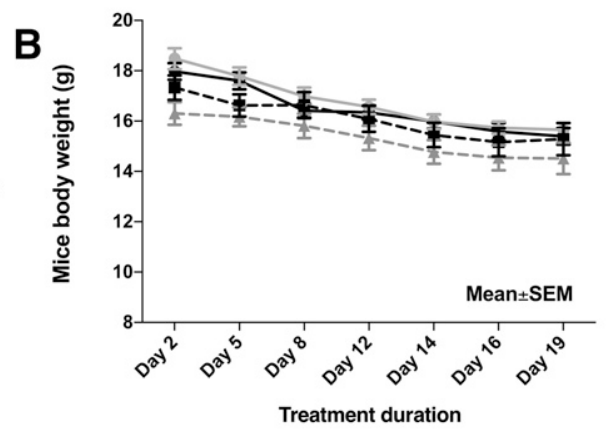
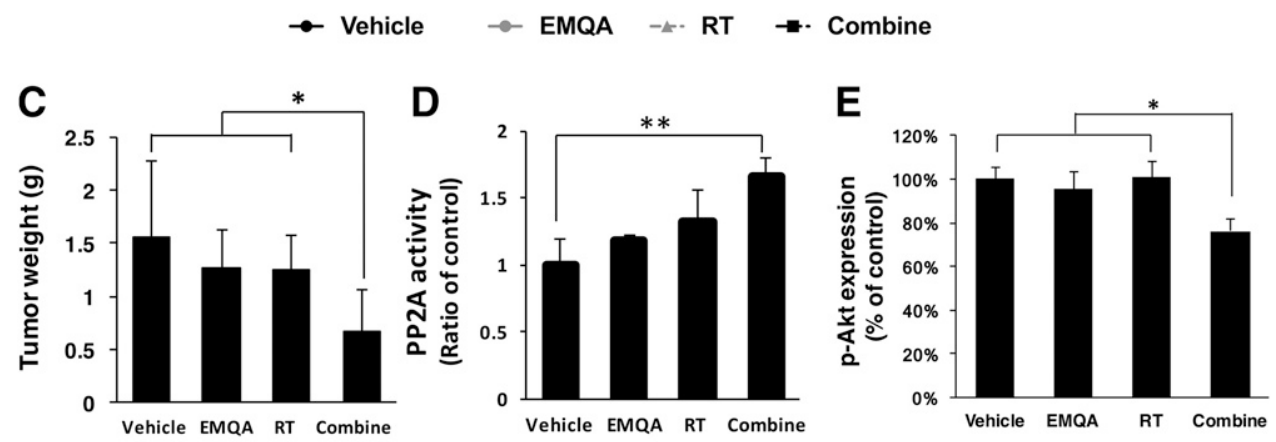

$\mathbf{F}$

Treatment

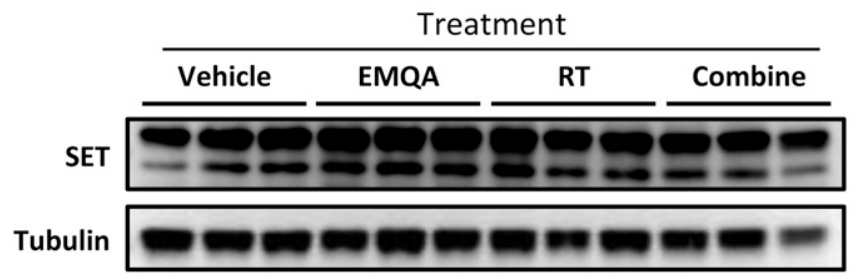

Fig. 6. EMQA synergized the anti-HCC effects of RT in vivo. (A) Combination treatment with EMQA and RT significantly inhibits in vivo HCC tumor growth. Mice with PLC5 xenografted tumors were treated with RT 5 Gy for 4 days and/or followed by daily EMQA treatment $(5 \mathrm{mg} / \mathrm{kg})$ when the size of tumor reached $500 \mathrm{~mm}^{3}$. The size of tumors was measured every other day during treatment and the growth curves of tumors were shown. Point, mean; error bars, S.E.M. ${ }^{*} P<0.05$. $(n \geq 7)$. (B) RT plus EMQA treatment shows acceptable tolerance. Weights of mice were monitored every other day during treatment. Point, mean; error bars, S.E.M. $(n \geq 7)$. (C) PLC5 xenografted tumors of mice in each treatment arm were resected and measured at the end of experiment. Bar, mean; error bar, S.D. $* P<0.05$. (D) PP2A activity of PLC5 xenograft tumor in vehicleand combination therapy-treated nude mice were shown in (D). Bar, mean; error bar, S.D. $* P<0.05$. (E and $\mathrm{F}$ ) The expression levels of p-Akt and SET in the PLC5 xenograft tumor lysate were analyzed by enzyme-linked immunosorbent assay (E) and Western blot $(\mathrm{F})$. Bar, mean; error bar, S.D. $* P<0.05, * * P<0.01$. oncogenic properties of SET (Li et al., 1996; Carlson et al., 1998; Switzer et al., 2011; Janghorban et al., 2014). Through direct interaction with the catalytic domain of PP2A, SET inactivates the tumor suppressor functions of PP2A and promotes aberrant activation of downstream oncogenic signaling, including AKT (Switzer et al., 2011). AKT is a key player in diverse cellular functions, such as proliferation, survival, and metabolism, and has been an attractive target for the design and development of novel anticancer treatments (Nitulescu et al., 2016). Several different AKT inhibitors are currently undergoing preclinical and early-stage clinical investigation (Lamoureux and Zoubeidi, 2013; Konopleva et al., 2014; Nitulescu et al., 2016). In this work, we presented a new approach, i.e., targeting AKT signaling in cancer via antagonizing SET-mediated PP2A inactivation, and showed the anticancer efficacy of this strategy in combination with RT. Since there are currently no active trials testing the efficacy of an AKT inhibitor plus RT, our data may provide rationale for future investigation.

We demonstrated that the use of RT alone exerted certain anti-HCC activity, and SET-knockdown or the additive use of a SET antagonist were able to further enhance the effects of RT against HCC. Regarding the use of RT for HCC, a fundamental challenge is the coexistence of underlying liver injury and hepatic dysfunction, which gives rise to increased concern about RT-induced liver complications (Hawkins and Dawson, 2006). However, such concern can now be largely dismissed owing to current advanced RT technology. With more powerful tools, such as breathing motion management, image guided radiation therapy, and stereotactic body RT, radiation oncologists today have more opportunities to deliver high and precise doses of radiation to target the liver tumor effectively, and avoid unwanted toxicity (Klein and Dawson, 2013). Certainly, the roles and the best dose-response relationship for RT in HCC have not yet been established. Additionally, biologic mechanisms that may diminish the effects of RT against HCC, such as the SET dysregulation we demonstrated in this work, still need to be explored fully. With increased investigation into these issues, we may improve the outcome in HCC patients by providing a personalized RT-integrated treatment to eliminate RT-related resistance and toxicity.

In conclusion, this study demonstrated the impact of the oncoprotein SET on the effects of RT therapy for HCC. Furthermore, through reactivation of PP2A-mediated p-AKT downregulation, a novel SET antagonist, EMQA, enhanced the effects of RT for the treatment of HCC in vitro and in vivo. Our data provide new insight into the oncogenic properties of SET, and show further that targeting SET/PP2A/p-AKT may be a feasible and effective strategy to enhance the antitumor effects of RT in HCC.

\section{Acknowledgments}

The authors acknowledge the service provided by the Flow Cytometric Analyzing and Sorting Core of the First Core Laboratory, National 
Taiwan University College of Medicine. They thank the National RNAi Core Facility at Academia Sinica in Taiwan for providing shRNA reagents and related services. The authors also thank the staff of the imaging core at the First Core Laboratories, National Taiwan University College of Medicine, for technical assistance. The authors especially acknowledge Dr. Pei-Yi Chu of Department of Pathology, Show Chwan Memorial Hospital, for Immunohistochemical (IHC) staining consultation.

\section{Authorship Contributions}

Participated in research design: Hung, K.-F. Chen.

Conducted experiments: Huang, Shih, Hsieh, Kuo, Tsai, Chang,

Hsiao, L.-J. Chen.

Performed data analysis: Huang, Hung, K.-F. Chen.

Wrote or contributed to the writing of the manuscript: Hung, Chao.

\section{References}

Adachi Y, Pavlakis GN, and Copeland TD (1994) Identification and characterization of SET, a nuclear phosphoprotein encoded by the translocation break point in acute undifferentiated leukemia. J Biol Chem 269:2258-2262.

Agarwal A, MacKenzie RJ, Pippa R, Eide CA, Oddo J, Tyner JW, Sears R, Vitek MP, Odero MD, Christensen DJ, and Druker BJ (2014) Antagonism of SET using OP449 enhances the efficacy of tyrosine kinase inhibitors and overcomes drug resistance in myeloid leukemia. Clin Cancer Res 20:2092-2103.

Al-Murrani SW, Woodgett JR, and Damuni Z (1999) Expression of I2PP2A, an inhibitor of protein phosphatase 2A, induces c-Jun and AP-1 activity. Biochem $J$ 341 293-298.

Arif M, Wei J, Zhang Q, Liu F, Basurto-Islas G, Grundke-Iqbal I, and Iqbal K (2014) Cytoplasmic retention of protein phosphatase 2A inhibitor 2 (I2PP2A) induces Alzheimer-like abnormal hyperphosphorylation of Tau. J Biol Chem 289: 27677-27691.

Arnaud L, Chen S, Liu F, Li B, Khatoon S, Grundke-Iqbal I, and Iqbal K (2011) Mechanism of inhibition of PP2A activity and abnormal hyperphosphorylation of tau by I2(PP2A)/SET. FEBS Lett 585:2653-2659.

Bonner JA, Harari PM, Giralt J, Cohen RB, Jones CU, Sur RK, Raben D, Baselga J, Spencer SA, Zhu J, et al. (2010) Radiotherapy plus cetuximab for locoregionally advanced head and neck cancer: 5 -year survival data from a phase 3 randomised trial, and relation between cetuximab-induced rash and survival. Lancet Oncol 11 $21-28$

Canela N, Rodriguez-Vilarrupla A, Estanyol JM, Diaz C, Pujol MJ, Agell N, and Bachs O (2003) The SET protein regulates G2/M transition by modulating cyclin B-cyclin-dependent kinase 1 activity. J Biol Chem 278:1158-1164.

Carlson SG, Eng E, Kim EG, Perlman EJ, Copeland TD, and Ballermann BJ (1998) Expression of SET, an inhibitor of protein phosphatase $2 \mathrm{~A}$, in renal development and Wilms' tumor. J Am Soc Nephrol 9:1873-1880.

Cervoni N, Detich N, Seo SB, Chakravarti D, and Szyf M (2002) The oncoprotein Set/ TAF-1 $\beta$, an inhibitor of histone acetyltransferase, inhibits active demethylation of DNA, integrating DNA methylation and transcriptional silencing. $J$ Biol Chem 277:25026-25031.

Chae H, Lim J, Kim M, Park J, Kim Y, Han K, Lee S, and Min WS (2012) Phenotypic and genetic characterization of adult T-cell acute lymphoblastic leukemia with $\operatorname{del}(9)(q 34) ;$ SET-NUP214 rearrangement. Ann Hematol 91:193-201.

Chen KF, Liu CY, Lin YC, Yu HC, Liu TH, Hou DR, Chen PJ, and Cheng AL (2010) CIP2A mediates effects of bortezomib on phospho-Akt and apoptosis in hepatocellular carcinoma cells. Oncogene 29:6257-6266.

Chen KF, Pao KC, Su JC, Chou YC, Liu CY, Chen HJ, Huang JW, Kim I, and Shiau CW (2012) Development of erlotinib derivatives as CIP2A-ablating agents independent of EGFR activity. Bioorg Med Chem 20:6144-6153.

Corvò R (2007) Evidence-based radiation oncology in head and neck squamous cell carcinoma. Radiother Oncol 85:156-170.

Cristóbal I, Garcia-Orti L, Cirauqui C, Cortes-Lavaud X, García-Sánchez MA, Calasanz MJ, and Odero MD (2012) Overexpression of SET is a recurrent event associated with poor outcome and contributes to protein phosphatase $2 \mathrm{~A}$ inhibition in acute myeloid leukemia. Haematologica 97:543-550.

Cristobal I, Rincon R, Manso R, Carames C, Zazo S, Madoz-Gurpide J, Rojo F, and Garcia-Foncillas J (2015) Deregulation of the PP2A inhibitor SET shows promising therapeutic implications and determines poor clinical outcome in patients with metastatic colorectal cancer. Clini Cancer Res 21:347-356.

El-Serag HB (2011) Hepatocellular carcinoma. N Engl J Med 365:1118-1127.

Facciuto ME, Koneru B, Rocca JP, Wolf DC, Kim-Schluger L, Visintainer P, Klein KM, Chun H, Marvin M, Rozenblit G, et al. (2008) Surgical treatment of hepatocellular carcinoma beyond Milan criteria. Results of liver resection, salvage transplantation, and primary liver transplantation. Ann Surg Oncol 15: 1383-1391.

Fan Z, Beresford PJ, Oh DY, Zhang D, and Lieberman J (2003) Tumor suppressor NM23-H1 is a granzyme A-activated DNase during CTL-mediated apoptosis, and the nucleosome assembly protein SET is its inhibitor. Cell 112:659-672.

Forner A, Llovet JM, and Bruix J (2012) Hepatocellular carcinoma. Lancet $\mathbf{3 7 9}$ $1245-1255$

Fukukawa C, Shima H, Tanuma N, Ogawa K, and Kikuchi K (2000) Up-regulation of I-2(PP2A)/SET gene expression in rat primary hepatomas and regenerating livers. Cancer Lett 161:89-95.

González-Arzola K, Díaz-Moreno I, Cano-González A, Díaz-Quintana A, VelázquezCampoy A, Moreno-Beltrán B, López-Rivas A, and De la Rosa MA (2015) Structural basis for inhibition of the histone chaperone activity of SET/TAF-I $\beta$ by cytochrome c. Proc Natl Acad Sci USA 112:9908-9913.
Hawkins MA and Dawson LA (2006) Radiation therapy for hepatocellular carcinoma: from palliation to cure. Cancer 106:1653-1663.

Huang CY, Lin CS, Tai WT, Hsieh CY, Shiau CW, Cheng AL, and Chen KF (2013) Sorafenib enhances radiation-induced apoptosis in hepatocellular carcinoma by inhibiting STAT3. Int J Radiat Oncol Biol Phys 86:456-462.

Hung M-H and Chen K-F (2017) Reprogramming the oncogenic response: SET protein as a potential therapeutic target in cancer. Expert Opin Ther Targets 21: $685-694$

Hung MH, Chen YL, Chu PY, Shih CT, Yu HC, Tai WT, Shiau CW, and Chen KF (2016) Upregulation of the oncoprotein SET determines poor clinical outcomes in hepatocellular carcinoma and shows therapeutic potential. Oncogene 35: $4891-4902$

Hung MH, Wang CY, Chen YL, Chu PY, Hsiao YJ, Tai WT, Chao TT, Yu HC, Shiau $\mathrm{CW}$, and Chen KF (2015) SET antagonist enhances the chemosensitivity of nonsmall cell lung cancer cells by reactivating protein phosphatase $2 \mathrm{~A}$. Oncotarget 7 : 638-655.

Huyen Y, Zgheib O, Ditullio RA Jr, Gorgoulis VG, Zacharatos P, Petty TJ, Sheston EA, Mellert HS, Stavridi ES, and Halazonetis TD (2004) Methylated lysine 79 of histone H3 targets 53BP1 to DNA double-strand breaks. Nature 432:406-411.

Janghorban M, Farrell AS, Allen-Petersen BL, Pelz C, Daniel CJ, Oddo J, Langer EM, Christensen DJ, and Sears RC (2014) Targeting c-MYC by antagonizing PP2A inhibitors in breast cancer. Proc Natl Acad Sci USA 111:9157-9162.

Kalousi A, Hoffbeck AS, Selemenakis PN, Pinder J, Savage KI, Khanna KK, Brino L, Dellaire G, Gorgoulis VG, and Soutoglou E (2015) The nuclear oncogene SET controls DNA repair by KAP1 and HP1 retention to chromatin. Cell Reports 11: 149-163.

Klein J and Dawson LA (2013) Hepatocellular carcinoma radiation therapy: review of evidence and future opportunities. Int J Radiat Oncol Biol Phys 87:22-32.

Końca K, Lankoff A, Banasik A, Lisowska H, Kuszewski T, Góźdź S, Koza Z, and Wojcik A (2003) A cross-platform public domain PC image-analysis program for the comet assay. Mutat Res 534:15-20.

Konopleva MY, Walter RB, Faderl SH, Jabbour EJ, Zeng Z, Borthakur G, Huang X, Kadia TM, Ruvolo PP, Feliu JB, et al. (2014) Preclinical and early clinical evaluation of the oral AKT inhibitor, MK-2206, for the treatment of acute myelogenous leukemia. Clin Cancer Res 20:2226-2235.

Kutney SN, Hong R, Macfarlan T, and Chakravarti D (2004) A signaling role of histone-binding proteins and INHAT subunits pp32 and Set/TAF-Ibeta in integrating chromatin hypoacetylation and transcriptional repression. $J$ Biol Chem 279:30850-30855.

Lamoureux F and Zoubeidi A (2013) Dual inhibition of autophagy and the AKT pathway in prostate cancer. Autophagy 9:1119-1120.

Li M, Guo H, and Damuni Z (1995) Purification and characterization of two potent heat-stable protein inhibitors of protein phosphatase $2 \mathrm{~A}$ from bovine kidney. Biochemistry 34:1988-1996.

Li M, Makkinje A, and Damuni Z (1996) The myeloid leukemia-associated protein SET is a potent inhibitor of protein phosphatase 2A. J Biol Chem 271:11059-11062.

Liu H, Gu Y, Wang H, Yin J, Zheng G, Zhang Z, Lu M, Wang C, and He Z (2015) Overexpression of PP2A inhibitor SET oncoprotein is associated with tumor progression and poor prognosis in human non-small cell lung cancer. Oncotarget 6 : 14913-14925.

Mumby M (2007) PP2A: unveiling a reluctant tumor suppressor. Cell 130:21-24.

Muslimovic A, Ismail IH, Gao Y, and Hammarsten O (2008) An optimized method for measurement of gamma-H2AX in blood mononuclear and cultured cells. Nat Protoc 3:1187-1193.

Nitulescu GM, Margina D, Juzenas P, Peng Q, Olaru OT, Saloustros E, Fenga C, Spandidos DA, Libra M, and Tsatsakis AM (2016) Akt inhibitors in cancer treatment: the long journey from drug discovery to clinical use (Review). Int J Oncol 48:869-885. Oike T, Niimi A, Okonogi N, Murata K, Matsumura A, Noda S-E, Kobayashi D, Iwanaga M, Tsuchida K, Kanai T, et al. (2016) Visualization of complex DNA doublestrand breaks in a tumor treated with carbon ion radiotherapy. Sci Rep 6:22275

Perrotti D and Neviani P (2013) Protein phosphatase 2A: a target for anticancer therapy. Lancet Oncol 14:e229-e238.

Piao LS, Hur W, Kim TK, Hong SW, Kim SW, Choi JE, Sung PS, Song MJ, Lee BC, Hwang D, et al. (2012) CD133+ liver cancer stem cells modulate radioresistance in human hepatocellular carcinoma. Cancer Lett 315:129-137.

Poon RT, Fan ST, Lo CM, Liu CL, and Wong J (2002) Long-term survival and pattern of recurrence after resection of small hepatocellular carcinoma in patients with preserved liver function: implications for a strategy of salvage transplantation. Ann Surg 235:373-382.

Ragaz J, Olivotto IA, Spinelli JJ, Phillips N, Jackson SM, Wilson KS, Knowling MA Coppin CM, Weir L, Gelmon K, et al. (2005) Locoregional radiation therapy in patients with high-risk breast cancer receiving adjuvant chemotherapy: 20-year results of the British Columbia randomized trial. J Natl Cancer Inst 97:116-126.

Rincón R, Cristóbal I, Zazo S, Arpí O, Menéndez S, Manso R, Lluch A, Eroles P, Rovira A, Albanell J, et al. (2015) PP2A inhibition determines poor outcome and doxorubicin resistance in early breast cancer and its activation shows promising therapeutic effects. Oncotarget 6:4299-4314.

Ruvolo PP (2016) The broken "Off" switch in cancer signaling: PP2A as a regulator of tumorigenesis, drug resistance, and immune surveillance. BBA Clin 6:87-99.

Seo SB, McNamara P, Heo S, Turner A, Lane WS, and Chakravarti D (2001) Regulation of histone acetylation and transcription by INHAT, a human cellular complex containing the set oncoprotein. Cell 104:119-130.

Shimura T, Noma N, Oikawa T, Ochiai Y, Kakuda S, Kuwahara Y, Takai Y, Takahashi A, and Fukumoto M (2012) Activation of the AKT/cyclin D1/Cdk4 survival signaling pathway in radioresistant cancer stem cells. Oncogenesis 1:e12.

Switzer CH, Cheng RY, Vitek TM, Christensen DJ, Wink DA, and Vitek MP (2011) Targeting SET/I(2)PP2A oncoprotein functions as a multi-pathway strategy for cancer therapy. Oncogene 30:2504-2513.

Tepper J, Krasna MJ, Niedzwiecki D, Hollis D, Reed CE, Goldberg R, Kiel K, Willett C, Sugarbaker D, and Mayer R (2008) Phase III trial of trimodality therapy with 
cisplatin, fluorouracil, radiotherapy, and surgery compared with surgery alone for esophageal cancer: CALGB 9781. J Clin Oncol 26:1086-1092.

Torre LA, Bray F, Siegel RL, Ferlay J, Lortet-Tieulent J, and Jemal A (2015) Global cancer statistics, 2012. CA Cancer J Clin65:87-108.

von Lindern M, van Baal S, Wiegant J, Raap A, Hagemeijer A, and Grosveld G (1992) Can, a putative oncogene associated with myeloid leukemogenesis, may be activated by fusion of its $3^{\prime}$ half to different genes: characterization of the set gene. $\mathrm{Mol}$ Cell Biol 12:3346-3355.

Yarnold J (1997) Molecular aspects of cellular responses to radiotherapy. Radiother Oncol 44:1-7.

Yu HC, Hung MH, Chen YL, Chu PY, Wang CY, Chao TT, Liu CY, Shiau CW, and Chen KF (2014) Erlotinib derivative inhibits hepatocellular carcinoma by targeting CIP2A to reactivate protein phosphatase 2A. Cell Death Dis 5:e1359.
Zhang W, Cai J, Chen S, Zheng X, Hu S, Dong W, Lu J, Xing J, and Dong Y (2015) Paclitaxel resistance in MCF-7/PTX cells is reversed by paeonol through suppression of the SET/phosphatidylinositol 3-kinase/Akt pathway. Mol Med Rep 12: $1506-1514$

Address correspondence to: Dr. Kuen-Feng Chen, Department of Medical Research, National Taiwan University Hospital, No 7, Chung-Shan S Rd, Taipei, Taiwan. E-mail: kfchen1970@ntu.edu.tw; or Dr. Man-Hsin Hung, Division of Medical Oncology, Department of Oncology, Taipei Veterans General Hospital, No 201, Sec. 2, Shipai Rd, Taipei, Taiwan. E-mail: cindybeaty@gmail.com 